\title{
Single-cell transcriptomics reveals specific RNA editing signatures in the human brain
}

\author{
ERNESTO PICARDI, ${ }^{1,2}$ DAVID S. HORNER, ${ }^{3}$ and GRAZIANO PESOLE ${ }^{1,2}$ \\ ${ }^{1}$ Department of Biosciences, Biotechnology and Biopharmaceutics, University of Bari, 70126 Bari, Italy \\ ${ }^{2}$ Institute of Biomembranes and Bioenergetics, National Research Council, 70126 Bari, Italy \\ ${ }^{3}$ Department of Biosciences, University of Milan, 20133 Milan, Italy
}

\begin{abstract}
While RNA editing by A-to-I deamination is a requisite for neuronal function in humans, it is under-investigated in single cells. Here we fill this gap by analyzing RNA editing profiles of single cells from the brain cortex of living human subjects. We show that RNA editing levels per cell are bimodally distributed and distinguish between major brain cell types, thus providing new insights into neuronal dynamics.
\end{abstract}

Keywords: RNA editing; single cell; RNA-seq; transcriptome

\section{INTRODUCTION}

Deep-sequencing technologies have allowed the characterization of patterns of transcriptome variation across individuals and tissues at unprecedented resolution-facilitating substantial advances in our understanding of transcriptional regulation and post-transcriptional processing (Mele et al. 2015). Recent advances in whole-transcriptome amplification have permitted quantitative sequencing of the minute amounts of RNA residing in single cells, and offer a unique opportunity to investigate transcriptional heterogeneity within tissues or even cell types by circumventing the need to study bulk tissue populations that usually comprise thousands or millions of input cells (Shapiro et al. 2013). Among human tissues, the adult brain shows extremely high complexity, being composed of a variety of cell classes and subtypes. Microfluidic systems coupled with massive mRNA sequencing on dissociated human brain cortex enable the identification of all major neuronal, glial, and vascular cell types, and reveal distinctive transcriptome profiles undetectable in the ensemble tissue (Darmanis et al. 2015). Despite these advances, cell level variation in post-transcriptional processing steps such as RNA modification by adenosine to inosine (A-to-I) editing, which actively contributes to transcriptome and proteome complexity, remains under-investigated.

A-to-I RNA editing is widespread in humans, affecting coding and noncoding transcripts at thousands of sites, and has a plethora of biological effects depending on the RNA context modified. RNA editing alters codon identity,

Corresponding author: graziano.pesole@uniba.it

Article is online at http://www.rnajournal.org/cgi/doi/10.1261/rna 058271.116. Freely available online through the RNA Open Access option. creates or eliminates splice sites, and interferes with basepairing interactions within higher-order RNA structures (Nishikura 2016). A-to-I deamination is catalyzed by members of the adenosine deaminase (ADAR) family of enzymes that act on dsRNA and occurs mainly in the primate-specific Alu repetitive elements (Nishikura 2016). ADARs are extremely important for the maintenance of cell homeostasis as mouse null mutants develop epileptic seizures and die several weeks after birth (Higuchi et al. 2000; Horsch et al. 2011). In addition, dysregulated RNA editing levels at specific recoding sites have been linked with a variety of diseases including neurological or psychiatric disorders and cancer (Chen et al. 2013; Behm and Öhman 2016; Khermesh et al. 2016).

We recently profiled A-to-I editing at the genome-wide level in various bulk human tissues and confirmed its pervasive nature, detecting more than 3 million events (Picardi et al. 2015). Our comprehensive catalog indicates strong tissue specificity of RNA editing and reveals the brain to be the human tissue with the most specific modifications. Indeed, A-to-I editing is required for neuronal function as many targets are key mediators of synaptic signaling (Mattick and Mehler 2008; Behm and Öhman 2016).

To capture and characterize the complexity of RNA editing at single-cell resolution, we exploited existing single cell RNA sequencing (scRNA-seq) data from adult human cortex cells obtained from living subjects (Darmanis et al. 2015). Our study provides novel and exciting insights into neuronal plasticity and opens up the possibility to decipher yet unknown

(c) 2017 Picardi et al. This article, published in $R N A$, is available under a Creative Commons License (Attribution 4.0 International), as described at http://creativecommons.org/licenses/by/4.0/. 
molecular aspects of RNA editing in physiological as well as diverse neurological or neurodegenerative disorders.

\section{RESULTS AND DISCUSSION}

We explored cell inosinome profiles using a comprehensive and nonredundant collection of known RNA editing events, including A-to-I changes from our catalog (Picardi et al. 2015) as well as sites annotated in the RADAR database (Ramaswami and Li 2014). The data allowed the interrogation of more than 4.5 million positions per cell.

Initially we processed raw scRNAseq data from 331 cells, performing quality checks, adaptor trimming, and in silico rRNA depletion. Cleaned reads were aligned onto the reference genome using the STAR mapper. Next, we excluded cells with fewer than 1 million uniquely aligned reads and a mapping rate $<70 \%$, reducing the starting data set to 268 cells. On average, we analyzed 2.3 million reads per cell (Supplemental Table 1), calling RNA editing events using REDItools (Picardi and Pesole 2013) and retaining only sites supported by at least 10 independent reads.

The number of detected A-to-I events varied greatly among cell types and within each cell population, principally as a consequence of variation in sequencing depth (Supplemental Table 1). Indeed, the number of A-to-I changes per cell was strongly correlated with the number of uniquely aligned reads $(r=0.83, P=0.0)$ (Supplemental Fig. 1). Strikingly, RNA editing levels (proportions of reads supporting an editing event at each known editing site) for individual cells showed a bimodal distribution with peaks close to extreme values (0 and 1) (Fig. 1A). This observation was not an artifact resulting from the presence of PCR duplicate reads, as PCR duplication was globally low (affecting on average $10 \%$ of aligned reads) (Supplemental Fig. 2). Furthermore, raw and de-duplicated data sets shared, on average, $95 \%$ of candidate editing sites and by position, comparison of A-to-I levels showed a remarkable positive correlation $(r=0.9998, P=0.0)$ (Supplemental Fig. 3). After removal of PCR duplicates, A-to-I editing levels of single cells continued to exhibit an extreme bimodal distribution (Fig. 1B). However, when scRNA-seq reads were merged, mimicking an ensemble tissue, RNA editing levels displayed a classical unimodal distribution in which the majority of A-to-I editing levels was lower than 0.2 , as previously observed in six human tissues (including brain cortex) (Fig. 1C; Picardi et al. 2015). These observations suggest that the penetrance of editing at single sites in single brain cortex cells shows an "all or nothing" distribution and that the affected sites vary between single cells, an effect that is masked by the study of bulk tissues. The bimodal distribution of RNA editing levels was also recently shown for C-to-U editing levels in homogeneous populations of mice macrophages (Harjanto et al. 2016).

The vast majority of RNA editing sites detected here and in previous studies (Bazak et al. 2014a; Picardi et al. 2015) reside in Alu repetitive elements. To provide a more realistic estimate of global editing activity per cell, we calculated the Alu editing index (AEI) per cell because it represents the weighted average editing level across all expressed Alu sequences (PazYaacov et al. 2015). We grouped AEI values per cell type according to transcriptome profiling previously characterized (Darmanis et al. 2015) and found that each cell type population exhibited a peculiar AEI distribution in which astrocytes and neurons appeared as the most edited cell types (Fig. 2A). To confirm cell specificity of RNA editing, we performed a multidimensional scaling (MDS) analysis using Spearman correlation coefficients calculated by pairwise comparisons of RNA editing levels in major brain cell types. Strikingly, four clusters emerge, corresponding to astrocytes, neurons, oligodendrocytes, and OPCs (oligodendrocyte precursor cells); the overlap between the latter cell types indicating similar RNA editing profiles (Fig. 2B; Supplemental Fig. 4 including all cell types).

We also checked the expressed levels of ADAR enzymes in all cell types and found a very uneven distribution for $A D A R$ gene (also known as ADAR1), while ADARB1 (known as $A D A R 2$ ) was expressed at very low levels but a bit more in neurons (Supplemental Fig. 5). Interestingly, the ADARB2 gene, with questionable editing activity (Chen et al. 2000), was expressed at detectable levels only in neurons, oligodendrocytes, and hybrid cells (Supplemental Fig. 5). However,
A

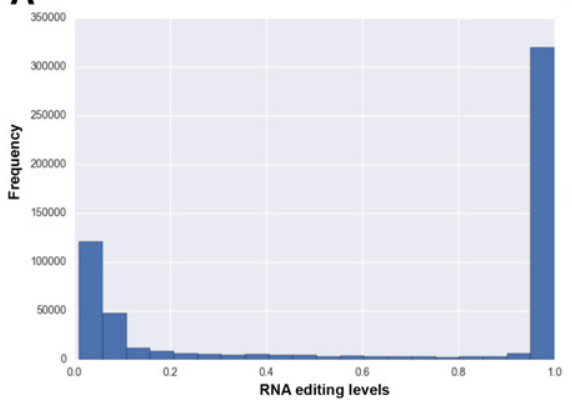

B

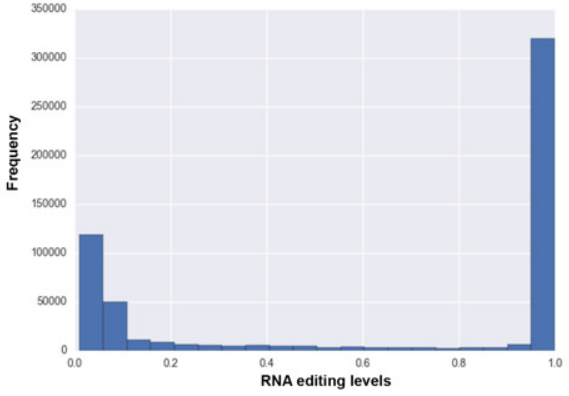

C

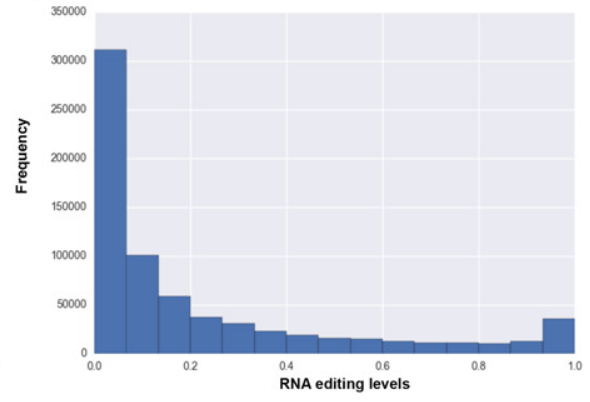

FIGURE 1. Distribution of RNA editing levels from all cells calculated with potential PCR duplicates $(A)$, without potential PCR duplicates $(B)$, and merging reads from all cells mimicking an ensemble tissue $(C)$. 


\section{Picardi et al.}
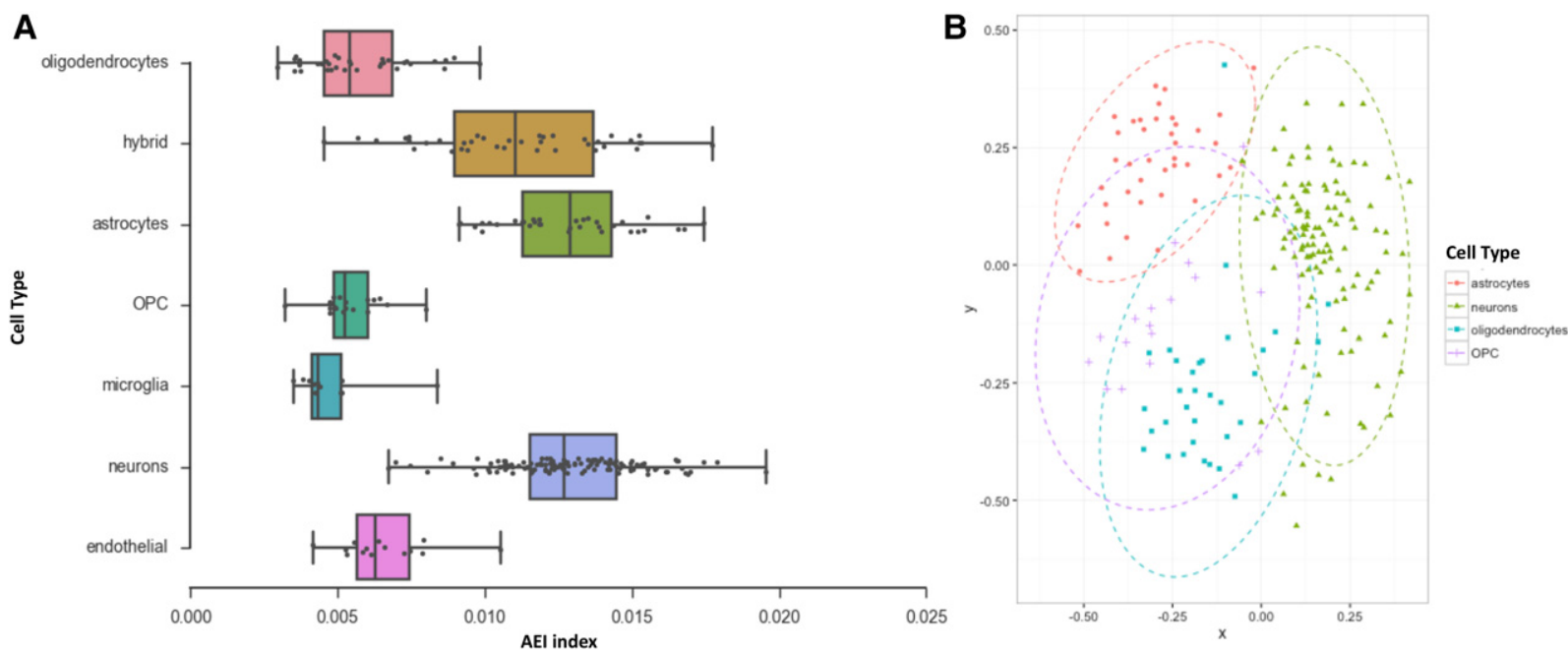

FIGURE 2. Distribution of AEI index across brain cell populations $(A)$ and MDS analysis of RNA editing profiles in major brain cell types $(B)$.

the expression levels of ADARs did not correlate with RNA editing levels and AEI values as well. In contrast, the AEI index showed strong correlation with the mean editing levels per cell (Spearman correlation value of $0.76, P$-value $=$ $\left.8.2 \times 10^{-53}\right)$ or the normalized sum of editing levels per cell (Spearman correlation value of $0.80, P$-value $=1.7 \times 10^{-61}$ ). The absence of significant correlation between the expression levels of $A D A R$ s and RNA editing levels or AEI values in single cells may be due to specific regulation mechanisms acting on ADAR enzymes or to a biased detection of gene expression levels. Indeed, many factors, such as cell cycle or cell size, may affect the correct quantification of gene expression levels, especially in cases of low expressed genes ( $\mathrm{Wu}$ et al. 2014; Kowalczyk et al. 2015; Padovan-Merhar et al. 2015). Additional experimental evidence will be required to clarify this aspect.

Historically, most interest has been directed toward recoding sites, where RNA editing results in amino acid substitutions. Many of the best-characterized recoding sites are in brain-specific transcripts coding for membrane receptors and ion channels (Behm and Öhman 2016). For 183 such positions selected from our editing collection and the RADAR database, RNA editing activity was higher in neurons than other cell types (Fig. 3). However, only a few sites, located in genes for glutamate receptors, were edited in almost all neurons, suggesting a universally critical role for editing at these sites (Supplemental Table 3). The data set used in this study also included a new hypothetical cell type indicated as "hybrid" that displayed characteristics of both neurons and astrocytes (Darmanis et al. 2015). The analysis of recoding sites indicated that hybrid cells were more similar to neurons than astrocytes (Fig. 3). "Hybrid" cells exhibited a recoding editing pattern of glutamate receptors that overlaps substantially with that observed in neurons (Fig. 3). In addition, MDS analysis of RNA editing profiles from "hybrid" cells, neurons, and astrocytes showed large overlap between "hy- brid" cells and neurons but not astrocytes (Supplemental Figs. 6, 7). Thus, our results support the view of "hybrid" cells as a potential new cell type related to neurons.

To further investigate the possibility that RNA editing profiles represent powerful signatures of cell type specificity, we analyzed single fetal brain cells, since they are considerably different from any cell type in the adult brain and because RNA editing efficiency increases during brain development and, consequently, different editing patterns are expected between fetal and adult cells (Wahlstedt et al. 2009). We interrogated 4.5 million known RNA editing positions in 135 scRNA-seqs from previously characterized fetal brain cells (Darmanis et al. 2015). They include 110 quiescent fetal neurons and 25 actively dividing fetal neuronal progenitors. AEI distributions clearly showed elevated editing activity in adult neurons compared to fetal neurons and higher editing in quiescent fetal neurons compared to fetal neuronal progenitors (Supplemental Fig. 8A). MDS analysis of RNA editing profiles confirmed three main groups, distinguishing adult from fetal neurons (Supplemental Fig. 8B). Notably, RNA editing activity at recoding sites was higher in adult than fetal neurons (Supplemental Fig. 9). In particular, the $\mathrm{Q} / \mathrm{R}$ site in Gria2, linked to neurological disorders, was edited to high levels in fetal quiescent neurons but not in neuronal progenitors as previously assessed in vitro (Pachernegg et al. 2015).

Taken together, our results demonstrate that RNA editing is detectable in single cells and demonstrates that A-to-I patterns reveal specific editing signatures distinguishing major cell types in the human brain. Profiling RNA editing in single cells may shed light on novel physiological roles of RNA editing in neuronal plasticity and in a variety of neurological/ neurodegenerative disorders. In addition, A-to-I changes in single cells may contribute to the identification of novel therapeutic targets or prognostic markers for innovative approaches of precision medicine. 


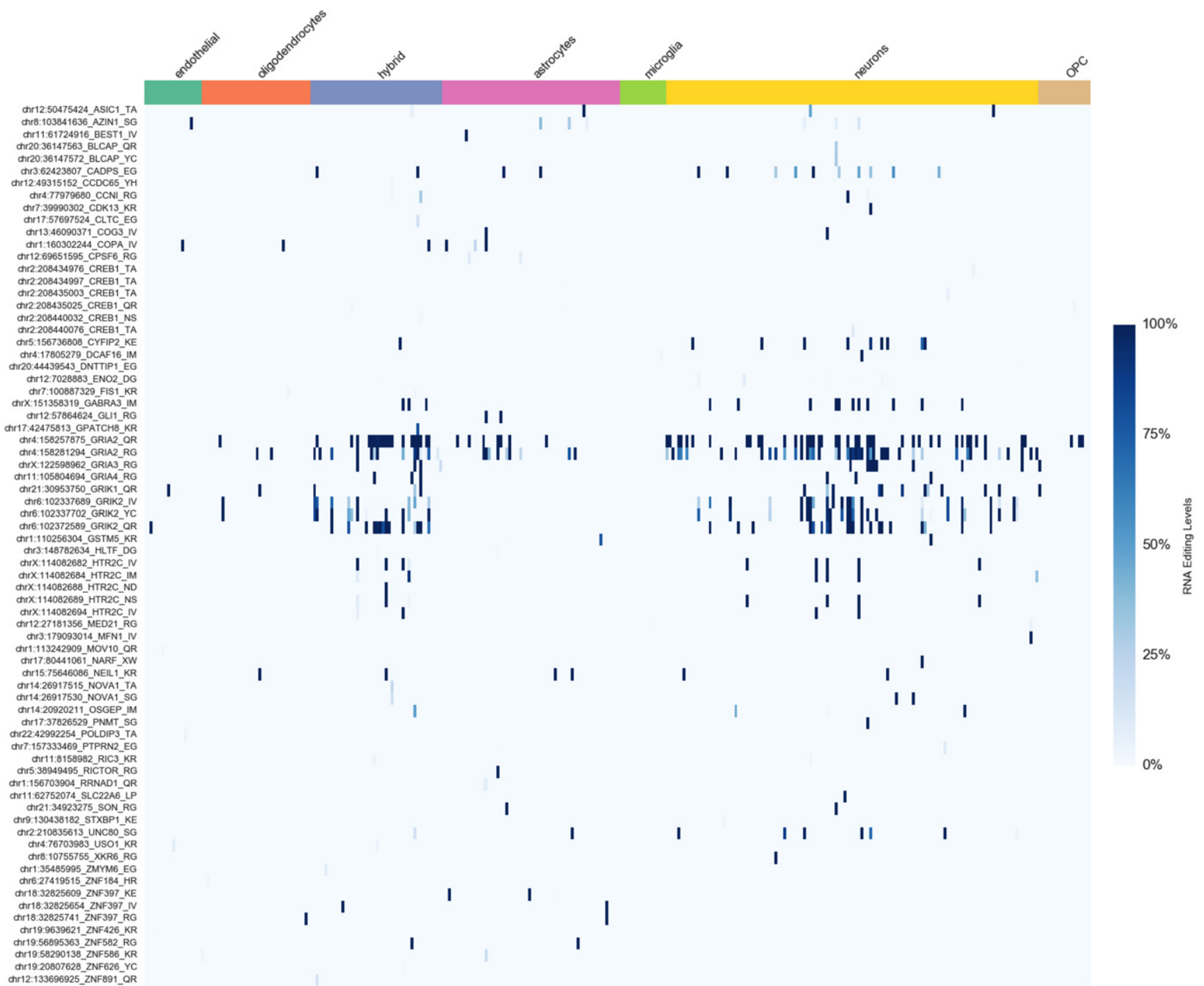

FIGURE 3. Heatmap of RNA editing levels at recoding sites in brain cells (see Supplemental Table 3 for details).

\section{MATERIALS AND METHODS}

\section{Data sets}

RNA-seq data of human brain single cells were downloaded from the Gene Expression Omnibus (GEO) database (www.ncbi.nlm. nih.gov/geo) using the accession number GSE67835. They comprise 332 cells from dissociated adult brain cortex and 134 cells from fetal brain tissue. Single cell capturing has been performed using Fluidigm C1 technology and RNA sequencing has been carried out on an Illumina NextSeq platform by Darmanis et al. (2015).

\section{Alignment of RNA-seq data}

RNA-seq reads in FASTQ format were inspected using the FASTQC program. Adaptors and low quality regions (phred cutoff of 25) were trimmed using TrimGalore (http://www.bioinformatics. babraham.ac.uk/projects/trim_galore/), excluding reads with final length less than 50 bases. In addition, we removed read pairs display- ing positive alignments with known human rRNA sequences. In brief, human rRNA annotations were downloaded from UCSC (hg19 human assembly) and indexed for STAR. Next, RNA reads were aligned onto rRNAs by STAR and only unmapped reads were retained for downstream analyses.

For each cell, cleaned reads were aligned onto the complete UCSC hg19 human genome by means of STAR (using as main parameters: -outSAMstrandField intronMotif -outFilterType BySJout -outFilterMultimapNmax 1 -alignSJoverhangMin 8 -alignSJDBoverhangMin 1 -outFilterMismatchNmax 999 -outFilterMismatchNoverLmax 0.04 -alignIntronMin 20 -alignIntronMax 1000000 -alignMatesGapMax 1000000).

Unique and concordant alignments in BAM format were processed by the CollectRnaSeqMetrics.jar tool from the Picard package to obtain basic statistics (Supplemental Table 1). Cells with fewer than 1 million uniquely aligned reads and a mapping rate $<70 \%$ were excluded from further analyses.

Duplicated reads were removed using the MarkDuplicates.jar tool from the Picard package. Since duplication was very low and did not 
affect RNA editing levels, we performed all RNA editing analyses on RNA-seq data with duplicates.

\section{RNA editing calling and data analysis}

In the absence of a whole-genome data set, we explored RNA editing profiles per cell comparing RNA-seq data with a comprehensive and nonredundant collection of known events, including A-to-I changes from our catalog (Picardi et al. 2015) as well as sites annotated in the RADAR database (Ramaswami and Li 2014). In all, we interrogated more than 4.5 million positions per cell using our REDItools suite. The complete collection is freely available through the REDIportal database (Picardi et al. 2016).

For each cell, REDItoolDnaRna.py script was called using the following parameters: $-\mathrm{c}$ 0,0 -T ALLediting.sorted.gtf.gz $-\mathrm{p}-\mathrm{u}-\mathrm{v} 0$ -n0 -G ALLediting.sorted.gtf.gz -e -m20,20 -q30,30. REDItool tables were finally parsed to retain only edited positions supported by at least 10 reads.

To measure the global editing activity per cell, we calculated the Alu editing index, the weighted average editing level across all expressed Alu sequences, using custom scripts and according to the methodology described in Bazak et al. (2014b).

RNA editing in recoding sites was assessed using REDItools and provided a list of 183 known positions in which RNA editing causes amino acid change. Such positions, listed in Supplemental Table 3, have been selected from our RNA editing Atlas as well as the RADAR database, picking only sites residing in nonrepetitive genomic regions and following criteria already described in Khermesh et al. (2016). As an exception, we included a NARF recoding site residing in an Alu region, since that site has already been validated in the literature (Moller-Krull et al. 2008).

Main statistical analyses and plots were performed using pandas, scipy, and matplotlib modules in python. Multidimensional scaling (MDS) was carried out in R using the metaMDS function of the vegan package, providing as input a Spearman correlation matrix calculated from editing levels for each cell type. Two-dimensional images depicting MDS clusters were generated by ggplot2.

\section{SUPPLEMENTAL MATERIAL}

Supplemental material is available for this article.

\section{ACKNOWLEDGMENTS}

We thank Ben Barres at Stanford University for helpful discussions about human brain cells and Eli Eisenberg at Tel Aviv University for assistance with Alu editing index computation. This work has been supported by the Flagship InterOmics (PB05) project, the Italian Ministero dell'Istruzione, Università e Ricerca (MIUR): PRIN 2012, and the Consiglio Nazionale delle Ricerche: Flagship Project Epigen, Medicina Personalizzata and Aging Program. Publication costs for open access have been funded by the Flagship InterOmics project.

Received July 11, 2016; accepted February 21, 2017.

\section{REFERENCES}

Bazak L, Haviv A, Barak M, Jacob-Hirsch J, Deng P, Zhang R, Isaacs FJ, Rechavi G, Li JB, Eisenberg E, et al. 2014a. A-to-I RNA editing occurs at over a hundred million genomic sites, located in a majority of human genes. Genome Res 24: 365-376.

Bazak L, Levanon EY, Eisenberg E. 2014b. Genome-wide analysis of Alu editability. Nucleic Acids Res 42: 6876-6884.

Behm M, Öhman M. 2016. RNA editing: a contributor to neuronal dynamics in the mammalian brain. Trends Genet 32: 165-175.

Chen CX, Cho DS, Wang Q, Lai F, Carter KC, Nishikura K. 2000. A third member of the RNA-specific adenosine deaminase gene family, ADAR3, contains both single- and double-stranded RNA binding domains. RNA 6: 755-767.

Chen L, Li Y, Lin CH, Chan TH, Chow RK, Song Y, Liu M, Yuan YF, Fu L, Kong KL, et al. 2013. Recoding RNA editing of AZIN1 predisposes to hepatocellular carcinoma. Nat Med 19: 209-216.

Darmanis S, Sloan SA, Zhang Y, Enge M, Caneda C, Shuer LM, Hayden Gephart MG, Barres BA, Quake SR. 2015. A survey of human brain transcriptome diversity at the single cell level. Proc Natl Acad Sci 112: 7285-7290.

Harjanto D, Papamarkou T, Oates CJ, Rayon-Estrada V, Papavasiliou FN, Papavasiliou A. 2016. RNA editing generates cellular subsets with diverse sequence within populations. Nat Commun 7: 12145.

Higuchi M, Maas S, Single FN, Hartner J, Rozov A, Burnashev N, Feldmeyer D, Sprengel R, Seeburg PH. 2000. Point mutation in an AMPA receptor gene rescues lethality in mice deficient in the RNA-editing enzyme ADAR2. Nature 406: 78-81.

Horsch M, Seeburg PH, Adler T, Aguilar-Pimentel JA, Becker L, Calzada-Wack J, Garrett L, Gotz A, Hans W, Higuchi M, et al. 2011. Requirement of the RNA-editing enzyme ADAR2 for normal physiology in mice. J Biol Chem 286: 18614-18622.

Khermesh K, D'Erchia AM, Barak M, Annese A, Wachtel C, Levanon EY, Picardi E, Eisenberg E. 2016. Reduced levels of protein recoding by A-to-I RNA editing in Alzheimer's disease. RNA 22: 290-302.

Kowalczyk MS, Tirosh I, Heckl D, Rao TN, Dixit A, Haas BJ, Schneider RK, Wagers AJ, Ebert BL, Regev A. 2015. Single-cell RNA-seq reveals changes in cell cycle and differentiation programs upon aging of hematopoietic stem cells. Genome Res 25: 1860-1872.

Mattick JS, Mehler MF. 2008. RNA editing, DNA recoding and the evolution of human cognition. Trends Neurosci 31: 227-233.

Mele M, Ferreira PG, Reverter F, DeLuca DS, Monlong J, Sammeth M, Young TR, Goldmann JM, Pervouchine DD, Sullivan TJ, et al. 2015. Human genomics. The human transcriptome across tissues and individuals. Science 348: 660-665.

Moller-Krull M, Zemann A, Roos C, Brosius J, Schmitz J. 2008. Beyond DNA: RNA editing and steps toward Alu exonization in primates. $J$ Mol Biol 382: 601-609.

Nishikura K. 2016. A-to-I editing of coding and non-coding RNAs by ADARs. Nat Rev Mol Cell Biol 17: 83-96.

Pachernegg S, Munster Y, Muth-Kohne E, Fuhrmann G, Hollmann M. 2015. GluA2 is rapidly edited at the Q/R site during neural differentiation in vitro. Front Cell Neurosci 9: 69.

Padovan-Merhar O, Nair GP, Biaesch AG, Mayer A, Scarfone S, Foley SW, Wu AR, Churchman LS, Singh A, Raj A. 2015. Single mammalian cells compensate for differences in cellular volume and DNA copy number through independent global transcriptional mechanisms. Mol Cell 58: 339-352.

Paz-Yaacov N, Bazak L, Buchumenski I, Porath HT, DananGotthold M, Knisbacher BA, Eisenberg E, Levanon EY. 2015. Elevated RNA editing activity is a major contributor to transcriptomic diversity in tumors. Cell Rep 13: 267-276.

Picardi E, Pesole G. 2013. REDItools: high-throughput RNA editing detection made easy. Bioinformatics 29: 1813-1814.

Picardi E, Manzari C, Mastropasqua F, Aiello I, D'Erchia AM, Pesole G. 2015. Profiling RNA editing in human tissues: towards the inosinome Atlas. Sci Rep 5: 14941. 
Picardi E, D’Erchia AM, Lo Giudice C, Pesole G. 2016. REDIportal: a comprehensive database of A-to-I RNA editing events in humans. Nucleic Acids Res 45: D750-D757.

Ramaswami G, Li JB. 2014. RADAR: a rigorously annotated database of A-to-I RNA editing. Nucleic Acids Res 42: D109-D113.

Shapiro E, Biezuner T, Linnarsson S. 2013. Single-cell sequencing-based technologies will revolutionize whole-organism science. Nat Rev Genet 14: 618-630.
Wahlstedt H, Daniel C, Enstero M, Ohman M. 2009. Largescale mRNA sequencing determines global regulation of RNA editing during brain development. Genome Res 19: 978-986.

Wu AR, Neff NF, Kalisky T, Dalerba P, Treutlein B, Rothenberg ME, Mburu FM, Mantalas GL, Sim S, Clarke MF, et al. 2014 Quantitative assessment of single-cell RNA-sequencing methods. Nat Methods 11: 41-46. 

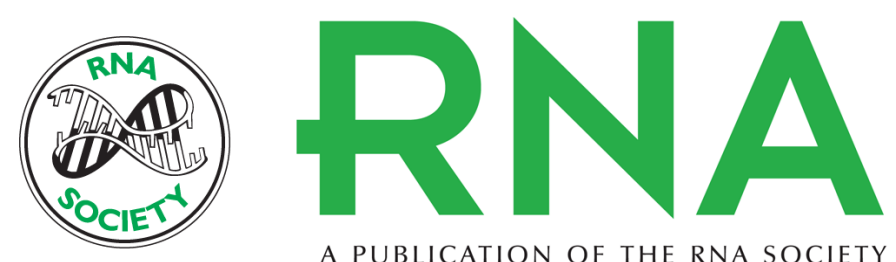

A PUBLICATION OF THE RNA SOCIETY

\title{
Single-cell transcriptomics reveals specific RNA editing signatures in the human brain
}

\author{
Ernesto Picardi, David S. Horner and Graziano Pesole
}

RNA 2017 23: 860-865 originally published online March 3, 2017

Access the most recent version at doi:10.1261/rna.058271.116

\section{Supplemental http://rnajournal.cshlp.org/content/suppl/2017/03/03/rna.058271.116.DC1 Material}

References This article cites 25 articles, 8 of which can be accessed free at: http://rnajournal.cshlp.org/content/23/6/860.full.html\#ref-list-1

Open Access Freely available online through the RNA Open Access option.

Creative This article, published in $R N A$, is available under a Creative Commons License Commons (Attribution 4.0 International), as described at

License http://creativecommons.org/licenses/by/4.0/.

Email Alerting Receive free email alerts when new articles cite this article - sign up in the box at the Service top right corner of the article or click here.

To subscribe to RNA go to:

http://rnajournal.cshlp.org/subscriptions

(C) 2017 Picardi et al.; Published by Cold Spring Harbor Laboratory Press for the RNA Society 